\section{Broken jaws in the COVID era}

Sir, we are already seeing the effects of a 'tsunami' of treatment need with a transference of most hospital resources to managing COVID-19 patients and a consequent diminution of resources for even the most urgent dental, medical and surgical conditions. ${ }^{1}$

One significant finding about the virus' behaviour is that health professionals, notably those working in ITU, dentistry, oral and maxillofacial surgery, ophthalmology and ENT, are particularly susceptible to severe infections which, in combination with a lack of protective equipment, reduces the active NHS workforce. This knowledge has been synthesised into position statements on NHS staff protection and working practice in the time of COVID-19. The British Oral and Maxillofacial (BAOMS) and oral surgeons (BAOS) have produced didactic guidance about risk and protection (https://www. baoms.org.uk).

At present there is no certainty about the pandemic timescale and the ability of NHS services to manage patients with COVID-19 and patients with other serious problems such as facial injuries. Also, in OMFS practice we must minimise cross infection from COVID patients to facial injury patients. The management of facial injuries has evolved over the last 100 years to our current evidence-based practice of anatomical reduction and internal fixation of complex facial injuries. This allows early mobilisation and rapid return to full jaw function, without the historical obligatory four to six weeks' rigid intermaxillary fixation (IMF) with wires.

However, cross-infection risk and the BAOMS and BAOS guidance about avoiding exposure, rapid hospital discharge, and simplifying and shortening surgical procedures has already resulted in abbreviated surgical treatment with older techniques such as IMF and potentially with follow-up delegated to the patient's dentist or doctor. ${ }^{2}$ There are three problems with GP or GDP follow up. Firstly, primary care practitioners must know how to manage the patient during the recovery period and remove intra-oral wires when the fractures have healed; they must also have the equipment and lighting to do this. They must also be remunerated satisfactorily for this work. Many dentists have closed their practices but it is likely that they will reopen once they are given proper protective equipment.

The wires around the teeth and jaws (IMF) remain until the jaw has healed. ${ }^{3}$ They make tooth cleaning difficult causing poor periodontal health. The GDP's weekly follow-up should include jaw fracture assessment and care of the periodontium. GDPs may need new skills and regular advice so we will provide learning and advice modules at: https://www.baoms.org.uk/ professionals/omfs_and_COVID-19.aspx.

\section{S. Holmes, I. Hutchison, D. Chatzopoulou,} London, UK

\section{References}

1. Hunter D J. COVID-19 and the stiff upper lip - the pandemic response in the United Kingdom. N Engl J Med 2020; DOI: 10.1056/NEJMp2005755.

2. Blitz M, Notarnicola K. Closed reduction of the mandibular fracture. Atlas Oral Maxillofac Surg Clin North Am 2009; 17: 1-13.

3. Thor A, Andersson L. Interdental wiring in jaw fractures: effects on teeth and surrounding tissues after a one-year follow-up. Br J Oral Maxillofac Surg 2001; 39: 398-401.

\section{https://doi.org/10.1038/s41415-020-1486-x}

\section{Telephone triaging and possible sepsis?}

Sir, the guidance from the Chief Dental Officer dated 25 March 2020 outlined changes in general dental and community practices with cessation of all routine, nonurgent dental care during this COVID-19 pandemic. ${ }^{1}$ The guidance informs that practices should provide a telephone triage service for advice, analgesia and antimicrobials (where appropriate) or refer patients to their Local Urgent Dental Care System for emergency dental care.

Telephone assessments will add a degree of complexity to patient assessment as visual signs will be lost. We wish to bring to attention the importance of vigilance for sepsis, one of the most common causes of death and disability in adults and children worldwide ${ }^{2}$ which can arise from a dental infection. Sepsis is defined as a lifethreatening organ dysfunction caused by a dysregulated host response to infection. ${ }^{3}$ Symptoms can vary but some hallmark signs include a core temperature $<36^{\circ} \mathrm{C}$ or $>38.5^{\circ} \mathrm{C}$, abnormal heart rate (tachycardia or bradycardia) or tachypnea. ${ }^{4,5}$ Altered mental state is another important red flag sign (such as new onset confusion, sleepiness, lethargy or agitation).

A high degree of vigilance is needed for patients who may have communication challenges such as those with learning disabilities, impaired consciousness and cognitive disorders. Dental practitioners may wish to devise a pro forma to ensure all relevant information is recorded during telephone consultations including a sepsis screen. Questions should include inquiry for local factors such as swelling, trismus, difficulty in breathing or swallowing and wider systemic factors such as hypothermia or fever, reduced urine output and changes in the skin such as cyanosis or non-blanching rashes.

May we please signpost readers to NICE clinical knowledge summaries, which provides useful information on sepsis diagnosis and management for patients seen in primary care? ${ }^{6}$

M. Dave, S. Barry, N. Patel, Manchester, UK

\section{References}

1. Hurley S, Neligan M. Preparedness-letter-forprimary-dental-care. United Kingdom: NHS England, 2020. Available at: https://www.england.nhs.uk/ coronavirus/wp-content/uploads/sites/52/2020/03/ issue-3-preparedness-letter-for-primary-dental-care-25march-2020.pdf (accessed 25 March 2020).

2. Wiens M O, Kumbakumba E, Kissoon N, Ansermino J M, Ndamira A, Larson C P. Pediatric sepsis in the developing world: challenges in defining sepsis and issues in postdischarge mortality. Clin Epidemiol 2012; 4: 319-325.

3. Singer M, Deutschman C S, Seymour C W et al. The Third International Consensus Definitions for Sepsis and Septic Shock (Sepsis-3). JAMA 2016; 315: 801-810.

4. Randolph A G, McCulloh R J. Pediatric sepsis: important considerations for diagnosing and managing severe infections in infants, children, and adolescents. Virulence 2014; 5: 179-189.

5. Goldstein B, Giroir B, Randolph A. International pediatric sepsis consensus conference: definitions for sepsis and organ dysfunction in pediatrics. Pediatr Crit Care Med 2005; 6: 2-8.

6. National Institute for Health and Care Excellence. Sepsis. United Kingdom: NICE, 2019. Available at: https://cks. nice.org.uk/sepsis (accessed 25 March 2020).

https://doi.org/10.1038/s41415-020-1487-9

\section{Education in infectious disease transmission}

Sir, it is widely accepted that dental schools need to teach curricula based on the principles of evidence-based dentistry. For many of us that instruction also includes teaching dental students the fundamentals of epidemiology and the basics of research methodology and study design. Increasingly, infectious disease outbreaks are receiving more attention due to the potential impacts of epidemics on an expanding global population and the fact that information in general is now disseminated much more rapidly than it was in 2003 when we had the Severe Acute Respiratory Syndrome (SARS) outbreak. 
Understanding how important outbreaks like SARS, Zika and most recently COVID-19, can spread so rapidly and their transmission curtailed should be of increasing concern for dental providers. In order to have comprehensive discussions with their patients about how infections can be transmitted in an epidemic, dental care providers need to be properly educated. However, very little time is allotted to instructing dental and dental hygiene students on public health approaches to interrupting disease transmission. Given these changes, dental educators should also focus on educating students on the most effective responses and interventions in controlling the spread of infectious disease. This could include an introduction to public health control measures such as environmental disinfection, facility closure, isolation of symptomatic cases, social distancing and health education, along with other approaches. Dental educators need to consider ways to formally incorporate instruction in best practices to prevent disease transmission and control of outbreaks into the dental curricula. In addition, we should also include online content that can provide the needed education to dental providers already in private practice. We have already started on developing these programmes.

B. Laurence, Washington, D.C., USA https://doi.org/10.1038/s41415-020-1488-8

\section{\#BeKind}

Sir, with a heavy heart I write to you to express how saddened and disappointed I am by the behaviour of some members of our profession. The COVID-19 pandemic has undoubtedly shaken and unsettled us all in every aspect of our lives. We are facing a global crisis unlike anything many of us will have ever faced before, and hopefully will never have to face again. Arguably, the pressure on our NHS has never been greater. These are truly difficult times for all. It is for that reason, that, for a moment, I felt ashamed to be part of what I considered to be an honourable profession, when I saw my fellow colleagues posting derogatory, defamatory and disparaging comments about our senior leaders on various online platforms.

COVID-19 is not only threatening our physical health but our mental health and livelihoods too, and naturally during such unprecedented times the profession will look to our senior leaders for guidance and reassurance. It is not for me to comment on the timeliness or effectiveness of such guidance, but I must say I find it appalling that we can call ourselves professionals, regulated and governed by professional standards of practice and behaviour, but participate in what is essentially 'internet trolling' or an online 'witch hunt' the moment uncertainty arises.

My call to arms for the profession is this: let this be the time that dentists and DCPs come together for the NHS to fight this battle with our medical and nursing colleagues. Let us use this opportunity to show just how powerful, committed and skilled we are as a profession. Do what you can in your regular clinical setting, and when you can no longer do that, do what you can in your community. Let's use this as an opportunity for NHS dentists and dentistry to be seen and treasured for the value it truly holds.

We are all under immense pressure, our leaders included, so let's do what we can, and above all \#BeKind.

F. Oluwajana, Manchester, UK https://doi.org/10.1038/s41415-020-1489-7

\section{NHS recruitment}

\section{Contract reform and funding}

Sir, the letter from Hussain, Thomson and Norfolk ${ }^{1}$ about recruitment and retention was an interesting read. For the past four years I have presented oral evidence to DDRB on this very concern, only to be faced with evidence submitted by NHS England and others that there is no problem, with 'dentists willing to tender for contracts'.

I agree with their summary that the worst problems in rural areas are extending across every English region, but the major reasons are not addressed in the letter. It is not that certain areas cannot encourage local school and college students into dentistry - indeed we have the highest number of dentists on the register perhaps of all time. The problems lie with the failing 2006 contract, the lack of flexibility within NHS England to adapt to support contractors and dentists struggling with the system, and the fact that my younger colleagues are no longer wedded to supporting a failing NHS dental service.

The applications at dental school remain remarkably buoyant and whilst it's always a good idea to go into educational establishments and encourage the potential dentists of the future, that alone will not resolve the recruitment and retention issues we see worsening.

East Anglia like the rest of England needs contract reform and a well-funded service for patients; that might solve the issues of recruitment and retention.

E. Crouch, Birmingham, UK

\section{Reference}

1. Hussain I, Thomson A, Norfolk T. Recruitment and retention. Br Dent J 2020; 228: 59.

https://doi.org/10.1038/s41415-020-1490-1

\section{Occupational health}

\section{Survival of the fittest}

Sir, overwhelming evidence, both clinical and anecdotal, affirms that dental professionals are especially vulnerable to musculoskeletal disorders (MSD), particularly those that affect the neck and back region. Literature continues to confirm that repetitive and prolonged actions, whilst employing a static, seated position, prove to be conducive to experiencing joint fatigue, stiffness or definitive pain. ${ }^{1}$ Such experiences are a universal concern for dental professionals and are greatly emphasised during undergraduate training.

Whilst undergraduate dental education involves learning about the complexities of the human body, seldom attention is placed on the complex interaction between our anatomy, mental state, and pain perception. Although $90 \%$ of back pain has a nonspecific cause, high rates are experienced amongst those with high stress levels or mental health concerns. ${ }^{2}$ Therefore, more attention needs to be given to the fact that such psychosocial factors can severely affect the risk of developing MSDs.

Indeed, the evolution of dental technologies has enhanced the practising experience for the dentist. Optimally fitted loupes in conjunction with the use of a saddle chair allows professionals to adopt a certain posture that allegedly reduces the risk of back pain. However, emerging evidence stipulates that the 'perfect posture' fails to actually exist. ${ }^{3}$ Therefore, it is critical that attention is directed towards various other strategies.

Weight management, improved mental state, and immune function are all beneficial consequences of regular physical activity. Therefore, physical exercise, particularly 